\title{
Investigation on the signal performances of pre-, post- and inline compensation of L- band dispersion compensator using chirped fiber Bragg grating
}

\begin{abstract}
The placement of Chirped Fiber Bragg grating in between the span plays an important role as it affects the signal quality when it reaches the transmission end. The work highlights on the signal performance at $10 \mathrm{Gbps}$ data rate after propagating through a $90 \mathrm{~km}$ transmission fiber. The worst BER is obtained in the inline compensation configuration as compared to pre- and post- compensation. The highest received power of $-0.19 \mathrm{dBm}$ with bit error rate of $2.65 \mathrm{E}-07$ is obtained in post-compensation configuration while the highest received power for postcompensation configuration is around $-0.7 \mathrm{dBm}$ with bit error rate of $4.95 \mathrm{E}-07$. However, pre-compensation configuration is chosen in further measurement as it gives the best power penalty as compared to other configurations when BER of 1 E-06 is used as the benchmark for the signal quality.
\end{abstract}

Keyword: CFBG; L-band; Pre-compensation; Post-compensation; Inline compensation 